\title{
Enhanced Oscillatory Activity in the Hippocampal-Prefrontal Network Is Related to Short-Term Memory Function after Early-Life Seizures
}

\author{
Jonathan K. Kleen, ${ }^{1}$ Edie X. Wu, ${ }^{1}$ Gregory L. Holmes, ${ }^{1}$ Rod C. Scott,,${ }^{1,2}$ and Pierre-Pascal Lenck-Santini ${ }^{1}$ \\ ${ }^{1}$ Department of Neurology, Neuroscience Center at Dartmouth, Dartmouth Medical School, Hanover, New Hampshire 03755, and ${ }^{2}$ UCL Institute of Child \\ Health, London WC1N 1EH, United Kingdom
}

\begin{abstract}
Neurological insults during development are associated with later impairments in learning and memory. Although remedial training can help restore cognitive function, the neural mechanisms of this recovery in memory systems are largely unknown. To examine this issue, we measured electrophysiological oscillatory activity in the hippocampus (both CA3 and CA1) and prefrontal cortex of adult rats that had experienced repeated seizures in the first weeks of life, while they were remedially trained on a delayed-nonmatch-to-sample memory task. Seizure-exposed rats showed initial difficulties learning the task but performed similarly to control rats after extra training. Whole-session analyses illustrated enhanced theta power in all three structures while seizure rats learned response tasks before the memory task. While performing the memory task, dynamic oscillation patterns revealed that prefrontal cortex theta power was increased among seizure-exposed rats. This enhancement appeared after the first memory-training steps using short delays and plateaued at the most difficult steps, which included both short and long delays. Further, seizure rats showed enhanced CA1-prefrontal cortex theta coherence in correct trials compared with incorrect trials when long delays were imposed, suggesting increased hippocampal-prefrontal cortex synchrony for the task in this group when memory demand was high. Seizure-exposed rats also showed heightened gamma power and coherence among all three structures during the trials. Our results demonstrate the first evidence of hippocampal-prefrontal enhancements following seizures in early development. Dynamic compensatory changes in this network and interconnected circuits may underpin cognitive rehabilitation following other neurological insults to higher cognitive systems.
\end{abstract}

\section{Introduction}

The remarkable ability of the nervous system to adapt to insults or deprivation is believed to originate from plasticity phenomena. Extensive training can restore functional deficits in the neocortex following sensory disruption in early development through compensatory neuroplasticity (Zhou and Merzenich, 2009; Merabet and Pascual-Leone, 2010). However, the mechanisms underlying restoration of higher cognitive functions such as learning and memory after neuronal insults (Markowitsch et al., 1985; Gathercole and Alloway, 2006) are still poorly understood, particularly within cortical-limbic networks.

Patients that experienced neurological insults during development often develop compensatory cognitive strategies that help circumvent functional deficits (Mateer et al., 1996; Stiles et al.,

\footnotetext{
Received May 2, 2011; revised Sept. 10, 2011; accepted Sept. 12, 2011.

Author contributions: J.K.K., E.X.W., G.L.H., R.C.S., and P.-P.L.-S. designed research; J.K.K. and E.X.W. performed research; J.K.K. and R.C.S. analyzed data; J.K.K., G.L.H., R.C.S., and P.-P.L.-S. wrote the paper.

This work was supported by the National Institutes of Health Grants (NIH) F30NS064624 (J.K.K.) and R21MH086833 (P.-P.L.-S.), the Emmory R. Shapses Research Fund; NIH Grants R01NS044295 and R01NS073083 (G.L.H.); and the Great Ormond Street Children's Charity (R.C.S.). We thank Peter Cuadrilla, Marcella Lucas, Forrest Miller, and Qian Zhao for assistance and support.

The authors declare no competing financial interests.

Correspondence should be addressed to Dr. Gregory L. Holmes, Dartmouth Medical School, 1 Medical Center Drive, Lebanon, NH 03756. E-mail: Gregory.L.Holmes@Dartmouth.edu.

DOI:10.1523/JNEUROSCI.2196-11.2011

Copyright $\odot 2011$ the authors $\quad 0270-6474 / 11 / 3115397-10 \$ 15.00 / 0$
}

1997). Subsequent cognitive improvements following a neurological insult are likely related to functional reorganization of neural circuits and adjustments in their dynamic coordination (Bach-y-Rita, 1990; Carmichael et al., 2001; Keller and Just, 2009). This plasticity can be measured on a network level in vivo through the quantification of oscillatory activity in local field potentials (Tsanov and Manahan-Vaughan, 2009; Voytek et al., 2010). Oscillations provide indices of ongoing neural processing among groups of neurons near the recording site (Mormann et al., 2005; DeCoteau et al., 2007; Wang, 2010). In particular, hippocampal theta $(4-12 \mathrm{~Hz})$ and gamma $(30-120 \mathrm{~Hz})$ oscillations in the hippocampus and prefrontal cortex (PFC) have been correlated with short-term memory processing in rats (Jones and Wilson, 2005; Montgomery and Buzsáki, 2007; Adhikari et al., 2010) with analogous findings in humans (Canolty et al., 2006; Rizzuto et al., 2006; Watrous et al., 2011). In turn, pathological disruption of the hippocampus produces altered oscillatory activity, which can parallel reduced cognitive ability (Chauvière et al., 2009; Marcelin et al., 2009; Adhikari et al., 2010; Sigurdsson et al., 2010; Voytek et al., 2010).

Given the critical role of the hippocampal-PFC network in short-term memory function, we examined whether memory training following a neurological insult during development produces neuroplastic changes in this system. We show that adult rats exposed to early-life seizures (ELSs) indeed demonstrate altered evolution of oscillation characteristics in the 
hippocampus and PFC during training for a memory task. Moreover, following remedial training these rats show enhanced levels of select theta and gamma theta oscillations during trials compared with controls, as well as a relationship between CA1-PFC coherence and accuracy. These differences may reflect an interstructure compensatory mechanism following neurological insults via hippocampal-PFC plasticity.

\section{Materials and Methods}

Animals and seizure model. Ten Sprague Dawley rats of either sex were born in-house, with half $(N=5)$ experiencing 100 flurothylinduced seizures during postnatal days $15-30$ (six to seven seizures per day). This procedure is a model of ELS in humans (Isaeva et al., 2006), with normal subsequent phenotypic development to adulthood and no spontaneous seizures or epileptiform abnormalities on electroencephalogram (EEG) recordings. Control rats $(N=5)$ did not receive seizures but were separated from the dam rat for the same amount of time. In adulthood (between postnatal days 100 and 120), all rats were implanted with electrodes in the CA3, CA1, and PFC on the right side. Following 1 week of surgical recovery, rats were food restricted to $85 \%$ of their free-feeding weights, after which behavioral training commenced with EEG recorded from the three structures continuously during all sessions (Fig. 1A,B). All animal procedures were approved by the Dartmouth Institutional Animal Care and Use Committee in accordance with National Institutes of Health guidelines, among conditions approved by the U.S. Department of Agriculture and the Association for Assessment and Accreditation of Laboratory Animal Care.

Surgery. Rats were anesthetized with isoflurane $(2-3 \%$ in oxygen) and custom tetrodes with $0.5 \mathrm{~mm}$ vertical span (to encompass the CA strata) were implanted in the dorsalintermediate hippocampal CA3 (Fig. 1C) (deepest tip at $4.5 \mathrm{~mm}$ lateral, $4.2 \mathrm{~mm}$ posterior, and $5.0 \mathrm{~mm}$ ventral from the bregma skull fissure) (Paxinos and Watson, 2005) and CA1 (Fig. 1D) (deepest tip at $3.0 \mathrm{~mm}$ lateral, 4.2 $\mathrm{mm}$ posterior, and $2.9 \mathrm{~mm}$ ventral). In addition, similar tetrodes with $1.5 \mathrm{~mm}$ vertical span (to encompass the prelimbic area) were implanted in the PFC (Fig. $1 E$ ) (deepest tip at 0.6 $\mathrm{mm}$ lateral, $3.2 \mathrm{~mm}$ anterior, and $4.6 \mathrm{~mm}$ ventral). Ground wires were soldered to bone screws, and reference wires were implanted in the cerebellum for each rat. All wires were plugged into an interconnect socket array (MILL-MAX) and encased in dental cement.

Electrophysiology. Electrodes consisted of four $25 \mu \mathrm{m}$ nichrome wires (California Fine Wire), held in a 25-gauge stainless steel guide tube (Small Parts Inc.). Operational amplifiers plugged directly to the rat connector (unity gain), and EEG signals were transmitted via a custom cable and a rotating commutator (Dragonfly Research \& Development Inc.), all referenced to the cerebellum electrode. Recordings were made in an operant chamber described previously (Kleen et al., 2010). During all sessions, EEG signals were recorded from two leads in each structure and amplified at $1000 \times$ gain before digitization at $2315 \mathrm{~Hz}$ (DigiData
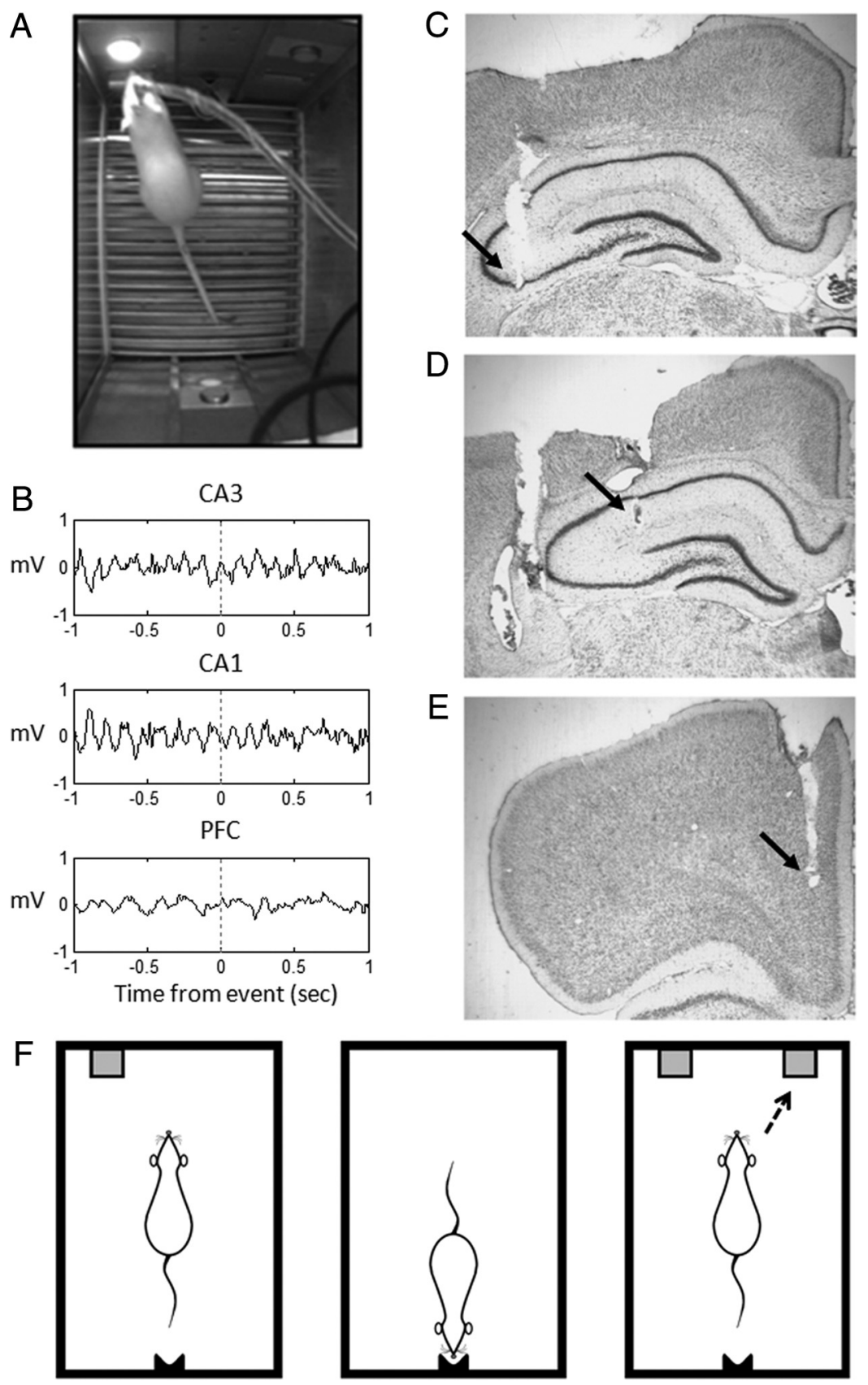

Figure 1. Behavior and electrophysiological recording. $\boldsymbol{A}$, Rat engaged in a DNMS trial, during the sample lever press. Depth EEG was recorded via a cable, which is visible in the picture. $\boldsymbol{B}$, Example EEG traces from each of the recorded areas in a control rat are shown around the moment of a sample lever press (dotted line). $C-E$, Representative thionin-stained coronal sections illustrating electrode placements in the CA3 $(\boldsymbol{C}), C A 1(\boldsymbol{D})$, and PFC (E). Arrows indicate the locations of the deepest electrode. $\boldsymbol{F}$, Outline of the DNMS task. The rat was required to press a sample lever ("sample"; left lever in this case), followed by an imposed delay period during which the rat had to break an infrared beam on the opposite wall. After the imposed delay the rat was required to press the opposite lever ("nonmatch"; right lever in this case, indicated by a dashed arrow) to gain a food reward. ture so that power and coherence analyses could be performed on the EEG signal from the most appropriately placed electrode, verified by histology before analysis.

Behavior. Rats sought food rewards in all tasks (45 mg Noyes food pellet; Research Diets Inc.). Training sessions were run at approximately the same time each day for each rat. Behavioral tasks that made up the training step sequence are listed in order on the $x$-axis in Figure $2 A$ and have been described previously (Brown and Jenkins, 1968; Kleen et al., 2010). The first step in response training was the "autoshape" step, which encouraged lever pressing by pairing the lever with a food reward (Brown 
A

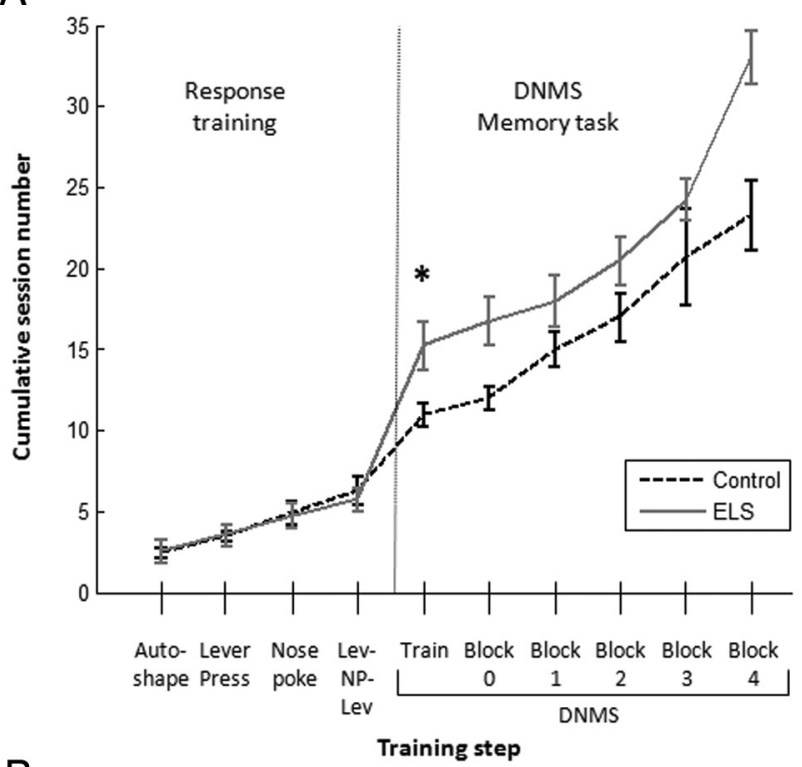

B

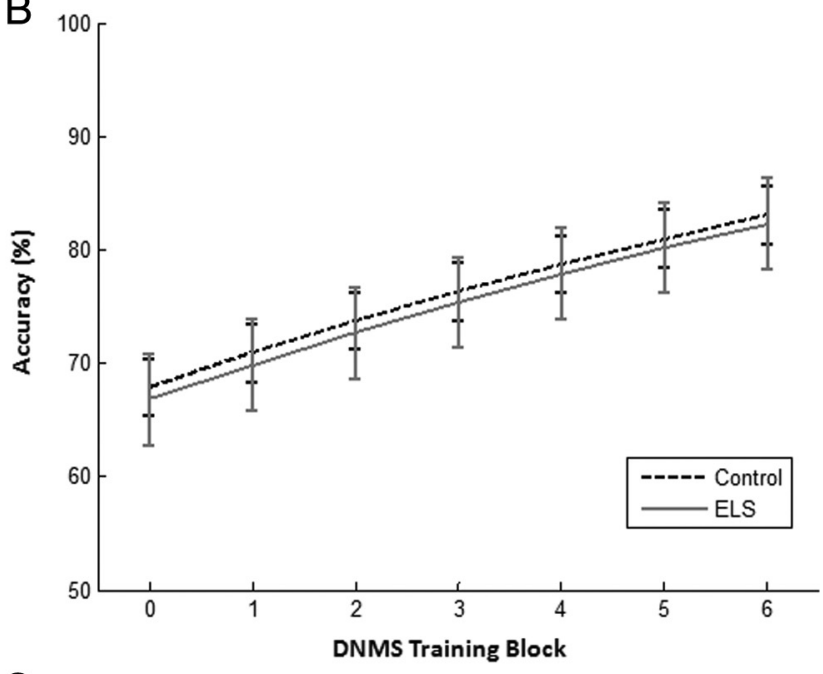

$\mathrm{C}$

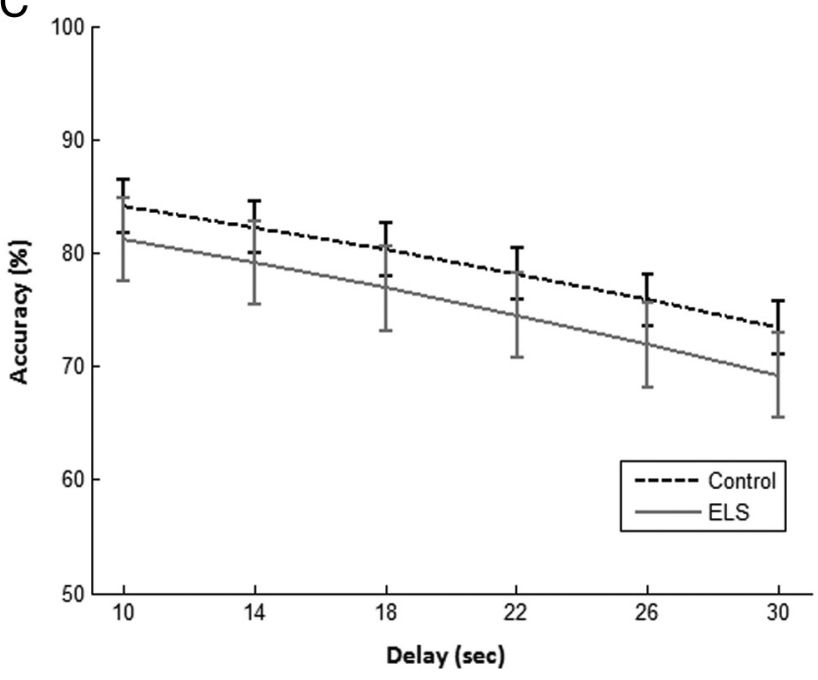

Figure 2. Behavioral performance. $A$, Learning progression during training, shown as the average cumulative session number ( \pm SEM) to reach criteria and advance to the next training step. Consecutive session types are listed on the $x$-axis. Because some rats did not complete criterion for all steps, Blocks 5 and 6 are not shown. Response training steps involved learning to press a lever for food reward (autoshape, lever press) and break an infrared beam (nosepoke) for food reward. The Lev-NP-Lev step involved an action sequence of pressing a randomly presented and Jenkins, 1968). Specifically, the lever was extended into the chamber and then retracted either after the rat pressed the lever or after $5 \mathrm{~s}$ had elapsed, followed by a food reward in both cases. This training step was followed by two fixed-ratio schedules, in which the rat was required to press the lever or break an infrared beam in the nosepoke apparatus to gain a food reward. The fourth step involved a sequence of levernosepoke-opposite lever to gain a food reward, preparing the rat for the action sequence of the upcoming delayed-nonmatch-to-sample (DNMS) task trials. Rats were required to gain a certain number of food rewards in a specified amount of time to advance to the next step, and these criteria were the same for all rats. Specifically, $>25$ responses within $45 \mathrm{~min}$ were needed in both the autoshape and lever-press steps, $>10$ beam breaks in $30 \mathrm{~min}$ for the nosepoke step, and $>40$ completed sequences in $45 \mathrm{~min}$ for the lever-nosepoke-opposite lever step.

All subsequent DNMS sessions were made up of 50 trials, lasting 45 min each, in which the rats were required to perform at least 40 trials correctly $(80 \%)$ to advance to the next training step. A DNMS trial (Fig. $1 F$ ) consisted of one lever (right or left) being randomly presented to the rat at first. Upon pressing the sample lever, the rat then had to break an infrared beam (nosepoke) on the opposite wall of the chamber. Immediately after the beam was broken, both levers were presented. A correct response was defined as pressing the lever that was not presented earlier as the sample (i.e., non-match-to-sample), which produced a food reward.

DNMS training sessions (Fig. $2 \mathrm{~A}$, DNMS train) began with a trial of DNMS, and if the animal performed it correctly, another was given, and so on. However, if the animal performed a trial incorrectly, the next trial was a lever-nosepoke-opposite lever trial (as in response training), followed immediately by another attempt at a DNMS trial. Following criterion performance ( $>80 \%$ successful trials), rats began DNMS-only sessions with no imposed delay at first (Fig. $2 \mathrm{~A}$, Block 0 ) followed by progressively increasing delay ranges. Delays were imposed by designating nosepokes as unproductive until a specified amount of time had elapsed after the sample press. This specified delay period was randomly selected in each trial, from a range that was defined for each DNMS block. The delay ranges for each block were as follows: Block 1, 1-5 s; Block 2, 1-10 s; Block 3, 1-15 s; Block 4, 1-20 s; Block 5, 1-25 s; Block 6, 1-30 s.

Data analysis. A repeated-measures ANOVA assuming a Poisson distribution for counts was used to evaluate the number of sessions taken to successfully complete the training steps. Accuracy and reaction time were analyzed using logistic regression adjusted for within-animal correlations, with each DNMS trial treated as an individual observation $(N=$ 5041 trials). Developmental and dynamic oscillation measures were analyzed using repeated-measures ANOVAs, with treatment and time as main effects, and an interaction term. Two ELS rats were not incorporated into oscillation analyses due to unreliable headstage connections. Sessions with substantial electrical noise (e.g., ground artifact) or in which rats showed clear preferences for one lever (i.e., nonmemory strategies) were not used in dynamic oscillation analyses.

Oscillation development. Power and coherence spectrograms were calculated with the entire EEG data traces from each behavioral session, using multitaper Fourier transform estimates on nonoverlapping windows ( $1 \mathrm{~s}$ for theta, $0.1 \mathrm{~s}$ for gamma). Custom software was written for these analyses using Matlab (MathWorks), built upon base software provided by Chronux (http://www.chronux.org) (Mitra and Bokil, 2008). Power spectrograms (time $\times$ frequency $\times$ power) were generated for power results within each brain area (CA3, CA1, and PFC), and coher-

$\leftarrow$

lever followed by nosepoke on the opposite wall, after which only the opposite lever from before was presented and had to be pressed. This prepared rats for the upcoming DNMS sequence in which both levers were presented after nosepoking to apply a memory component, as described in Figure $1 F$ and in the Materials and Methods section. ELS rats initially took longer to learn this task during the DNMS Train step $\left({ }^{*} p<0.01\right)$. B, Accuracy as a function of training block ( \pm SEM), modeled using logistic regression. Despite adding longer delays to each consecutive block, accuracy increased over the course of training with no significant difference between the groups. C, Accuracy as a function of delay ( \pm SEM; all blocks included), modeled using logistic regression. Accuracy decreased as delay increased. 

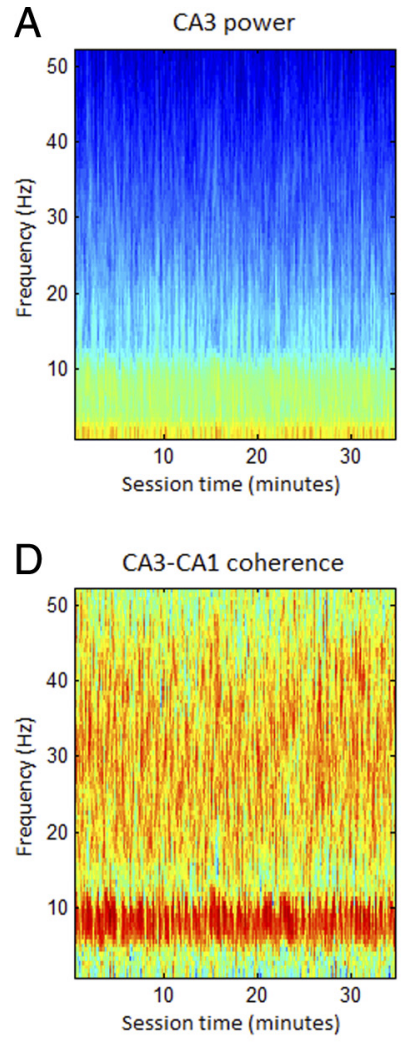
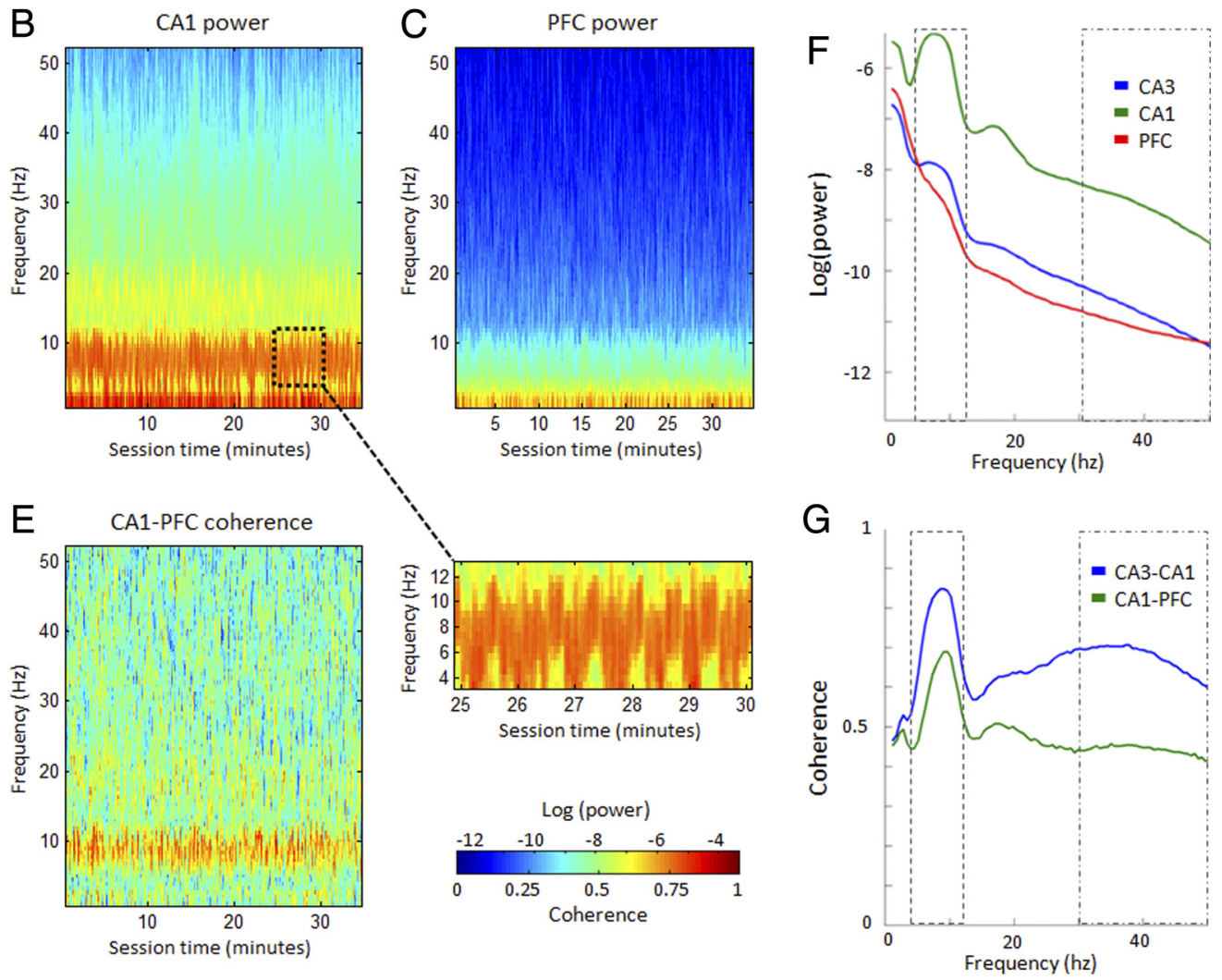

Figure 3. Examples of power and coherence spectra. $\boldsymbol{A}-\boldsymbol{E}$, Power $(\boldsymbol{A}-\boldsymbol{C})$ and coherence $(\boldsymbol{D}, \boldsymbol{E})$ spectrograms from a single DNMS session are depicted, using a $1 \mathrm{~s}$ nonoverlapping window. The slightly striated appearance reflects the performance of repeated DNMS trials over the entire session. The zoomed inset panel arising from the dotted window in $\boldsymbol{B}$ shows this more clearly over the course of a 5 min period, with a cyclic theta pattern corresponding to consecutive trials. Note the prominent theta (4-12 Hz) and gamma ( $30-50 \mathrm{~Hz})$ activity particularly in the $\mathbf{C A} 1$ power $(\boldsymbol{B})$ and CA3-CA1 coherence $(\boldsymbol{D})$ panels. $\boldsymbol{F}, \boldsymbol{G}$, Theta and gamma band peaks are better reflected in the median power $(\boldsymbol{F})$ and coherence $(\boldsymbol{G})$ spectra examples, calculated from the spectrograms in $\boldsymbol{A}-\boldsymbol{E}$. Note the peaks in theta power, which is highest in CA1. Theta coherence is prominent between all structures, and notable gamma coherence band is evident between CA3 and CA1 compared with CA1 and PFC.

ence spectrograms (time $\times$ frequency $\times$ coherence) were generated separately for CA3-CA1 signals and CA1-PFC signals. Frequency indices calculated from the initial window size were then used to extract the data within the theta and gamma bands separately. Resulting data within the active behavioral session (time locked to behavioral event signals from the chamber interface) were summed (power) or averaged (coherence) across frequency bands of $4-12 \mathrm{~Hz}$ for theta and $30-50 \mathrm{~Hz}$ for gamma. This produced a linear time vector for each measure over the length of the session with data points at $1 \mathrm{~s}$ intervals for theta and $0.1 \mathrm{~s}$ intervals for gamma. The medians of these vectors were taken as the general power and coherence values for that session. These values were then normalized by the values from the first session (the autoshape session) (see Fig. 4A, $C$, Behavior section in Materials and Methods, and group averages) to allow for visualization of changes from baseline over time.

Dynamic oscillation analysis. Continuous wavelet transforms were calculated on segments of EEG data (down-sampled by a factor of four to a $579 \mathrm{~Hz}$ sampling rate) that began $6 \mathrm{~s}$ before the sample press and ended $6 \mathrm{~s}$ after the match press for each trial. Custom software was written for these analyses using Matlab, built upon base software provided by Aslak Grinsted (Arctic Centre, University of Lapland, Rovaniemi, Finland) (Grinsted et al., 2004). Wavelet estimation allowed similar representation of power and coherence as time-frequency spectrograms, yet with time resolution equal to that of the raw data traces. The Morlet wavelet was used to provide a balance between time and frequency resolution. This wavelet function has been used in previous studies of theta and gamma oscillations (Montgomery and Buzsáki, 2007; Colgin et al., 2009). Specific features of the wavelet analysis used here are described by Grinsted et al. (2004). For each trial, wavelet power spectrograms were generated for the CA3, CA1, and PFC regions; coherence spectrograms were also generated for CA3-CA1 and CA1-PFC signals. Frequency in- dices calculated from period scaling factors were then used to extract the data within the theta and gamma bands. These data were summed (power) or averaged (coherence) across the data within the theta band frequencies and across the data within the gamma band frequencies $(30-50 \mathrm{~Hz})$. The resultant vectors were dynamic (real-time) time series of summed power or average coherence data, which was then smoothed using a $0.5 \mathrm{~s}$ (theta) or $0.2 \mathrm{~s}$ (gamma) moving average. To avoid edge estimations defined by the cone of influence in wavelet analyses (Grinsted et al., 2004), the first and last second of each data segment were removed. The resulting dynamic oscillation estimate for each trial was then down-sampled to $10 \mathrm{~Hz}$ for statistical analyses and normalized by the corresponding pretrial baseline. Only trials with delays between 10 and $30 \mathrm{~s}$ were used to ensure appropriate time allocation for encoding, maintenance, and retrieval processes (Kleen et al., 2010). Each rat's data were averaged across trials with delays between 10 and $30 \mathrm{~s}(N=3313$ trials) to adjust to equal weights for analysis. Data periods between -1.5 and $5 \mathrm{~s}$ around the sample press and -5 and $0 \mathrm{~s}$ before the match press were analyzed to encompass memory encoding, maintenance, and retrieval.

\section{Results}

Learning impairments and subsequent recovery in ELS rats Rats were trained on each step until criterion performance had been attained in a session (i.e., remedial training; see Materials and Methods), whereupon they advanced to the next training step. Learning abilities were thereby indexed by the number of sessions taken to advance through the consecutive training steps over time. The groups performed similarly during response training but ELS rats required significantly more DNMS Train 
A
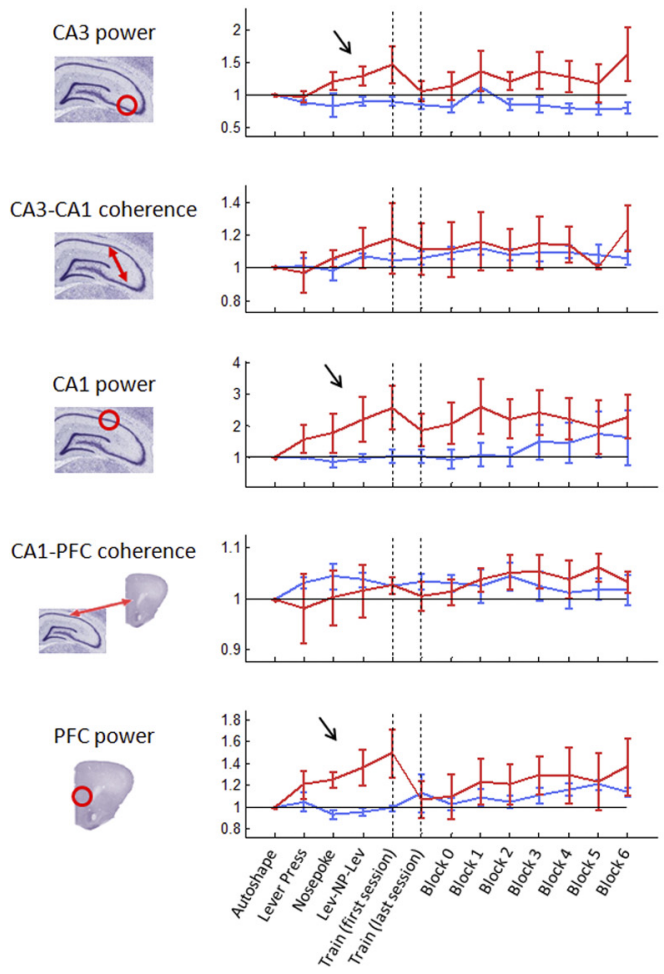

gamma") is specifically involved in CA3CA1 coordination (Colgin et al., 2009). Analysis of raw power (Fig. $3 A-C, F$ ) and coherence (Fig. $3 D, E, G$ ) revealed characteristic theta and gamma oscillation peaks in the CA3, CA1, and PFC (Colgin et al., 2009; Sigurdsson et al., 2010). Raw median power and coherence measures for oscillation power and coherence were calculated for each session using Fourierbased analyses and averaged across frequencies within the theta and gamma bands. These did not differ statistically between the groups in any of the structures during the first behavioral session except for CA3 gamma power $1(p<0.05)$. However, raw power and coherence can be influenced by small variations in electrode placement, and since the goal of our study was to assess changes in oscillation measures over the course of training (see Materials and Methods), we normalized all subsequent session values by the first training step to adjust for these potential differences.

By the end of response training, ELS animals showed robust increases in both theta power in the CA3, CA1, and PFC compared with controls $(p<0.001$ for all) (Fig. $4 A$ ). CA1 gamma power also increased in this group during this period while controls remained at baseline (Fig. $4 B$ ). Thus, ELS rats displayed atypically enhanced local oscillation power during

Figure 4. Evolution of whole-session power and coherence during training. $\boldsymbol{A}$, The progression of median theta power and coherence values across the progressive session training steps, averaged across rats in each group. ELS animals showed enhanced theta power in all structures during response training (solid arrows). B, Progression of median gamma power and coherence values across the session training steps. Control but not ELS animals showed an increase in CA3-CA1 coherence over the DNMS steps (dashed arrow).

sessions to achieve criterion performance $(p<0.01)$ (Fig. $2 A)$, in agreement with previous studies on ELS and hippocampaldependent task deficits (Holmes, 2009; Karnam et al., 2009a,b). Following eventual acquisition of the DNMS task ( $80 \%$ correct in DNMS Training; see Materials and Methods) via remedial training, ELS rats paralleled controls in learning the more difficult DNMS steps (Fig. $2 A$, blocks $0-4$ ). Data for Blocks 5 and 6 are not shown in Figure $2 \mathrm{~A}$ since some animals experienced electrode headstage malfunctions before passing criterion for those later blocks, skewing the group averages and SEs upon illustration. All animals increased in accuracy as training progressed over the consecutive DNMS blocks, with no difference in overall accuracy between the groups ( $p=0.58)$ (Fig. $2 B$ ) using logistic regression on 5041 trials adjusted for within-animal correlations. As in previous studies (Hampson et al., 1999; Kleen et al., 2010), delay was the main predictor of accuracy $(p<0.001$; odds ratio, 1.06; 95\% CI, 1.04-1.07) (Fig. 2C), with longer delays decreasing the likelihood of correct responses. Neither reaction time (the amount of time taken to press the match lever after it was extended into the chamber) nor the distribution of delays differed between ELS and control rats ( $p=0.14$ and $p=0.17$, respectively). Further, the relation between session step and time to complete a session did not differ between the groups $(p=0.15)$, indicating similar speeds and activity levels.

\section{Altered evolution of power and coherence in ELS rats during task acquisition}

We measured oscillation bands of $4-12 \mathrm{~Hz}$ for theta and $30-50$ $\mathrm{Hz}$ for gamma in this study because this latter range ("low response training (Pych et al., 2005).

As training progressed into the consecutive DNMS steps, control rats showed increased PFC theta power and decreased CA3 theta power $(p<0.001)$ (Fig. $4 A)$. Meanwhile, ELS animals maintained increases in both CA3 and CA1 theta power $(p<$ 0.001). Furthermore, while both groups showed increased gamma power in the CA1 and PFC (Fig. $4 B$ ), ELS rats also showed significantly increased CA3 gamma power $(p<0.001)$. A clear increase in CA3-CA1 gamma coherence across the DNMS steps in control rats diverged from that in ELS rats, which remained at baseline levels $(p<0.001)$. Meanwhile, ELS rats also developed a steady increase in CA1-PFC theta coherence that was not significant in controls ( $p<0.001)$ (Fig. $4 A)$. In other words, while changes in the intrahippocampal systems were observed in controls during DNMS training, ELS rats showed more general enhancement of the hippocampal-PFC network.

\section{Alternate dynamic oscillation patterns among ELS rats during DNMS trials}

Differences in general oscillation development during training suggest that alternate cognitive strategies may be emerging. To establish whether dynamic oscillation patterns during trials differed between the groups, we next examined dynamic power and coherence differences between the groups during trial performance using wavelet analysis. This provided a continuous temporal resolution ( $N=3313$ trials adjusted for individual rats) (Fig. 5). These measures were time locked to the sample and match presses, and normalized for each trial by dividing by a pretrial baseline ( -5 to $-2.5 \mathrm{~s}$ before the sample press) to adjust 


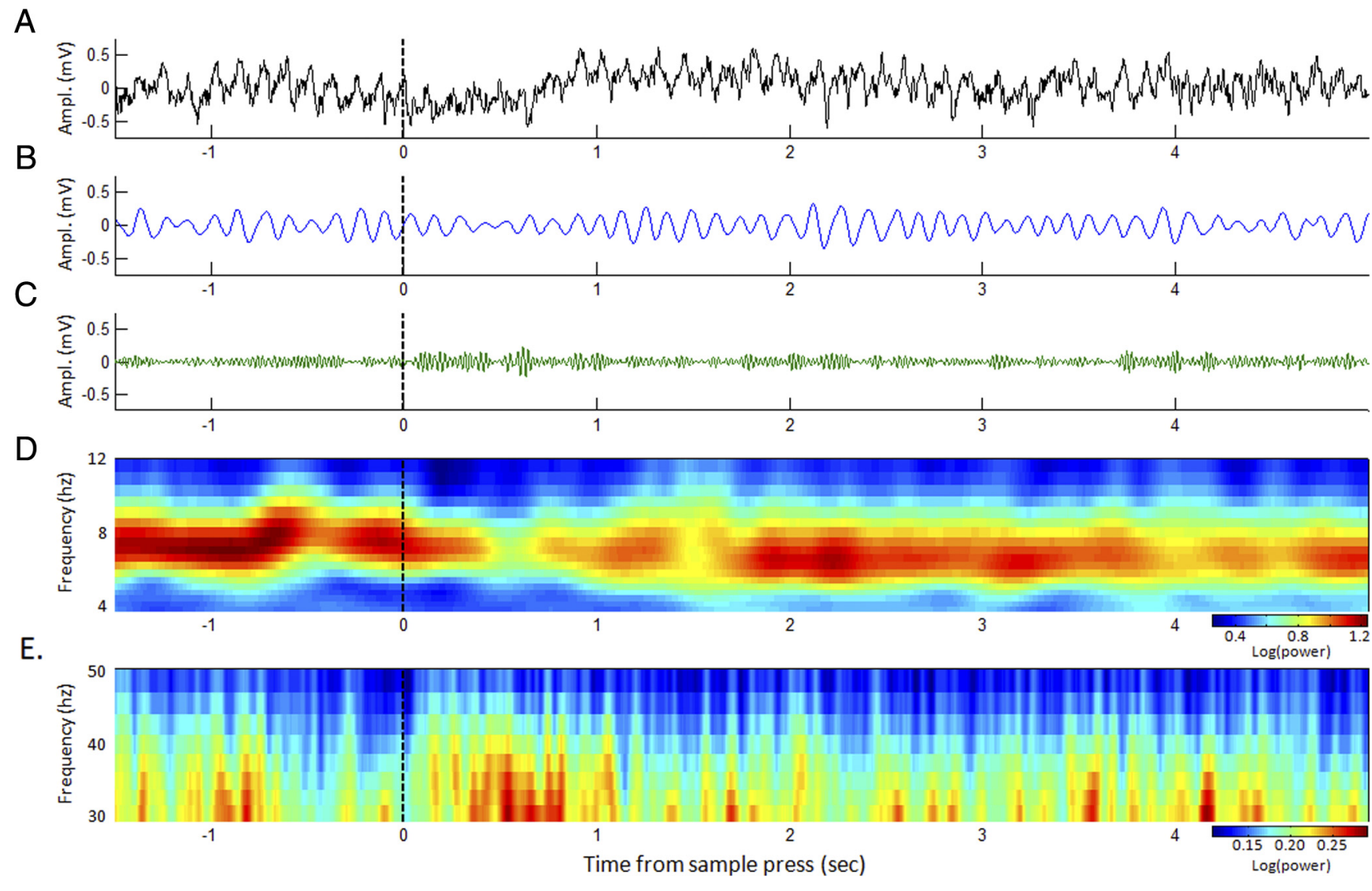

Figure 5. Dynamic oscillations during real-time performance. $A$, Example of CA1 $\mathrm{EEG} \mathrm{recorded} \mathrm{during} \mathrm{the} \mathrm{beginning} \mathrm{of} \mathrm{a} \mathrm{DNMS} \mathrm{trial} \mathrm{(dashed} \mathrm{line} \mathrm{indicates} \mathrm{the} \mathrm{moment} \mathrm{of} \mathrm{the} \mathrm{sample} \mathrm{lever} \mathrm{press).}$ $\boldsymbol{B}, \boldsymbol{C}$, The same data are shown filtered in the $4-12 \mathrm{~Hz}$ theta band $(\boldsymbol{B})$ and in the $30-50 \mathrm{~Hz}$ gamma band $(\boldsymbol{C})$. $\boldsymbol{D}, \boldsymbol{E}$, Averaged power spectrogram over all completed trials $(N=45)$ in a session by one rat (including the trial in $\boldsymbol{A}$ ) are also shown for frequencies in the theta band $(\boldsymbol{D})$ and gamma band $(\boldsymbol{E})$. Spectrograms were averaged within frequency bands in each trial to produce dynamic traces for the analyses in Figures $6-8$.

for initial differences between rats and across sessions, as evidenced in Figure 4. Pretrial baseline values for power and coherence did not show consistent changes over the course of training for any of the power or coherence measures.

The dynamic patterns of control animals in Figure $6 \mathrm{~A}$ (blue curves) illustrate the general functional modulation of theta oscillations in the DNMS task. Specifically, decreases in theta power and coherence among all structures were evident at the time of the sample press, followed by increased theta power in all structures immediately afterward as the rat turned to the other side of the chamber for the delay period ( $p<0.001$ for all, repeatedmeasures ANOVA adjusted within animal) (Fig. 6A). In the seconds preceding the match press, CA1-PFC theta coherence was persistently increased from pretrial baselines $(p<0.001)$ but returned to baseline at the end of the trial, confirming previous studies of short-term memory tasks in rats (Jones and Wilson, 2005; Sigurdsson et al., 2010). Decreases in gamma power and coherence were seen among nearly all structures before and after the sample press, and shortly before the match press $(p<0.001$ for all) (Fig. 7A).

We then examined divergences of ELS rat oscillation patterns (Fig. 6A, red curves) from controls. All ELS rats showed increases in PFC cortex power that began at the trial initiation, culminated to highest levels shortly before the match press $(p<0.001)$, and converged with controls after the trial. CA1-PFC theta coherence was also markedly increased compared with controls around the sample press $(p<0.001)$. Moreover, gamma power and coherence were increased among all structures in ELS animals com- pared with their control counterparts during the trials $(p<0.001$ for all) (Fig. 7A), returning to control levels immediately after trials were complete. These oscillatory activity enhancements among ELS rats during trials reveal heightened involvement and coordination of the hippocampal-PFC network during shortterm memory processing.

We then examined at which point these differences may have arisen during the course of training. Many of the biggest differences in dynamic oscillations between control and ELS animals occurred during the -4 and $-2 \mathrm{~s}$ preceding the match press (Fig. $6 \mathrm{~A}$, gray box) (i.e., around the time the animal had to recall which lever it had pressed previously). Thus, for each power and coherence measure we averaged this period to a single value in each trial (again normalized to the pretrial baseline), and then quantified how these levels changed over the course of training by grouping the DNMS training blocks into three consecutive levels-Blocks $0-1,2-3$, and $4-6$. Figure $6 B$ reveals that PFC theta power is similar between the groups in Blocks 0 and 1 and that it increases in ELS rats in Blocks 2 and 3, reaching its highest levels during Blocks 4, 5, and 6. A repeated-measures ANOVA showed an increasing trend among all rats $(p=0.056)$ and confirmed a treatment effect in this measure $(p<0.001)$. No other theta power or coherence measures were statistically significant ( $p>0.05$ for all), in agreement with the dynamic envelopes in Figure $6 \mathrm{~A}$.

We analyzed gamma evolution over time using the same time period during the trial (Fig. 7A, gray box). CA3 power (repeatedmeasures ANOVA, $p<0.05)$, CA3-CA1 coherence $(p<0.01)$, 


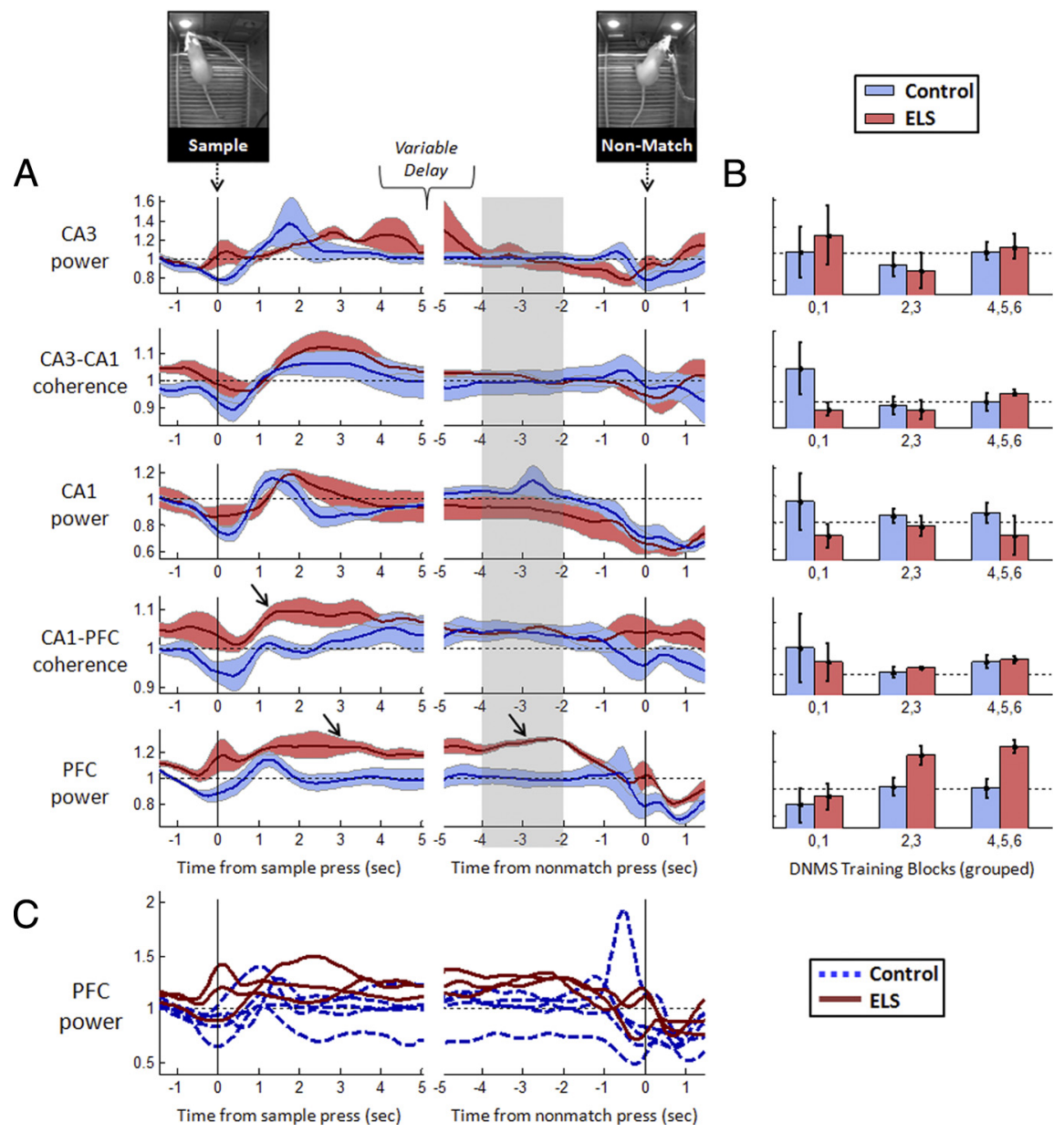

Figure 6. Dynamic theta power and coherence during real-time performance of DNMS trials. $\boldsymbol{A}$, Power and coherence were calculated within subject and are illustrated as colored envelopes bounded by the SEs around the means (solid lines). Data on left is time locked to the sample press, and data on the right to the match press (with variable delays between the two), revealing functional differences between control (blue) and ELS (red) rats when the envelopes diverge. ELS rats showed elevated PFC theta power from the beginning to the end of the trial (sample to match press) and increased CA1-PFC coherence following the sample press (solid arrows). $\boldsymbol{B}$, Evolution of theta in the retrieval period (gray box in $\boldsymbol{A}$ ) over the course of DNMS training, with training blocks grouped into three consecutive levels. C, PFC power data from individual rats used to produce the averaged data in $\boldsymbol{A}$ (last panel). Notice that all ELS rats showed consistently higher PFC power than controls in general, particularly before the match lever press.

CA1 power $(p<0.001)$, CA1-PFC coherence $(p<0.01)$, and PFC power $(p<0.05)$ were consistently elevated levels among ELS rats across all blocks (Fig. 7B). CA3-CA1 gamma coherence additionally showed a trend toward increasing levels over the training blocks $(p=0.057)$ when an interaction term for treatment versus block was factored into the statistical model.

We next examined the influence of trial difficulty on the EEG oscillation measures by analyzing the same data (Figs. 6A, $7 A$, gray boxes) grouped in delays of increasing duration: 10-15, 15-20, 20-25, and 25-30 s. Across all levels of delay, ELS animals showed significantly higher values of PFC theta power $(p<$ $0.001)$ and all gamma power and coherence measures $(p<0.01$ for all) compared with controls. However, no theta or gamma oscillation measures were significantly modulated by delay nor were any interactions present between delay and treatment group.

\section{CA1-PFC coherence predicted memory performance in ELS but not control rats}

To confirm the functional relevance of these oscillation patterns, we compared correct versus incorrect trials. In trials with longer delays (20-30 s), CA1-PFC coherence was markedly increased among correct trials relative to incorrect trials in ELS rats but not in controls (Fig. $8 A, B$ ). This trend was absent in both groups among short delays (10-20 s) (Fig. 8C), and other measures of theta and gamma power and coherence did not show this degree of performance modulation. These results indicate that ELS rats had increased hippocampal-PFC interplay when memory demand was high (Lee and Kesner, 2003).

\section{Discussion}

We showed that following a neurological insult early in life, the recovery of cognitive performance in adult rats is associated with the development of distinct patterns of brain oscillations in the hippocampalPFC network. ELS rats showed altered theta and gamma oscillations compared with controls, in both general development during task acquisition and dynamic activity patterns during task performance. Some of the dynamic measures were unequivocally observed in all ELS rats (Fig. 6C), suggesting a stereotyped neural adaptation to the insult.

ELS rats exhibited initial impairments relative to controls in learning the DNMS task as expected following this neurological insult (Holmes, 2009; Karnam et al., 2009a,b), but were able to perform as well as controls with increasing delays on the remedial training paradigm (Blocks 0-6). We first measured baseline power and coherence among all structures in the first session, noting similar values in all but CA3 power, which was lower among ELS animals. Although the recording sites were verified through postmortem histology, it is not clear whether a different baseline between the groups could be caused by ELS or was simply due to variations in electrode placement within the structures of interest. However, the goal of this study was to assess changes in EEG oscillation patterns over the course of training and during real-time performance of DNMS trials; thus, we used normalized power and coherence measures to allow visualization of these fluctuations.

We noted divergences of general levels of theta power in all structures during response learning and of CA3-CA1 gamma coherence during DNMS acquisition (Fig. $4 B, D$, respectively). These findings demonstrate that experience-dependent changes in oscillation coordination were emerging in ELS animals over the course of training. However, general averages in the wholesession analysis (Fig. 4) do not directly assess the functional activity pattern modifications that are critical for task performance. To examine whether the EEG activity patterns during real-time performance differed between the groups, we performed the dynamic analyses depicted in Figures 5-8.

We uncovered a number of group differences that suggested that the maintenance of performance in ELS animals may have arisen via compensatory processing in auxiliary networks. In par- 
ticular, ELS rats demonstrated large increases in PFC theta power, which developed in the more difficult DNMS training stages (Blocks 2-6). Additionally, all structures showed variable increases in gamma power and coherence that persisted during the task, implying stronger local information processing in these areas (Beshel et al., 2007; Colgin and Moser, 2010). Furthermore, enhanced CA1-PFC coherence was related to enhanced performance in the more difficult trials (longer delays). This effect was not seen in control rats, suggesting that ELS animals in particular may have relied upon increased hippocampal-PFC communication when the task demand was high.

Although we could not measure running speed due to confinements in the operant chamber, potential disparities in movement between groups likely imparted little to no influence on our results in part given equivalent measures of activity such as reaction time and time to complete sessions. Moreover, we noted similar group levels of dynamic CA1 theta power (Fig. 6A,B), which is known to be modulated by speed and movement (McFarland et al., 1975) in addition to learning and memory processing (Montgomery et al., 2009). Our interpretation and conclusions here relate mainly to findings regarding PFC theta power and CA1-PFC coherence, and we are not aware of literature clearly linking these measurements to movement or running speed similar to CA1 theta power.

Abnormal alterations in local networks can induce neuroplasticity to recruit auxiliary networks or enhance existing functional networks for effective computation (Feldman, 2009). Previous work shows that rats exposed to ELS have consistent alterations in hippocampal cellular and synaptic properties later in life that relate to cognitive dysfunction (de Rogalski Landrot et al., 2001; Cornejo et al., 2007). The ELS rats in this study showed disparate PFC-related theta oscillation patterns, suggesting that they may have adapted to faulty hippocampal circuitry by developing a more frontal lobe-reliant strategy (Lee and Kesner, 2003). When task demands required retrieval of memory after a longer delay, hippocampal interplay (i.e., CA1-PFC coherence) may have served to reconcile discrepant information (Wall and Messier, 2001). This compensatory possibility has been hypothesized in lesion studies of the hippocampus and PFC (Lee and Kesner, 2003; Wang and Cai, 2006), and we provide evidence here via dynamic electrophysiological activity profiles within this network during task performance.

The network mechanisms for the performance of delayedmatch and nonmatch paradigms have been examined in both animal and human settings. Lesion studies have emphasized the clear role of the hippocampal circuitry in these paradigms (Kirkby and Higgins, 1998; Hampson et al., 1999), as well as PFC contributions (Joel et al., 1997). Work using single-unit activity (Hampson et al., 1993; Hampson and Deadwyler, 1996) showed that neurons in the hippocampus respond selectively to events in the DNMS, such as specific phases of the task (sample vs press) or behavioral events (nosepoke or bar press). It has been shown that medial PFC neurons can be entrained to hippocampal theta frequency (Hyman et al., 2005; Siapas et al., 2005), and there is now strong evidence that this entrainment is involved in learning and memory in various working memory tasks (Jones and Wilson, 2005; Benchenane et al., 2010; Hyman et al., 2010). Kojima and Goldman-Rakic (1982) noted that PFC neurons exhibiting spatial-related firing properties tend to fire most strongly in the first few seconds of the delay period for a spatial delayed-response task. Perhaps in line with this, control rats in our study showed increased PFC theta at $\sim 1 \mathrm{~s}$ into the delay but then dropped back to pretrial baseline levels. ELS rats showed increased PFC theta (and CA1-PFC theta in correct trials with long delays) immediately after the sample press, and their levels did not return to baseline until $1 \mathrm{~s}$ before the match press. This implies that although the PFC may be important for short- or no-delay versions of DNMS under normal conditions, ELS rats engage this structure more persistently throughout trials. Together, these results demonstrate that increases in hippocampus and PFC coordination and PFC activity may support performance in the DNMS task after a neurological insult early in life.

Large increases of PFC theta power in ELS rats during the delay likely reflect more of an adjunct to hippocampal-mediated memory function, not a replacement. The PFC maintains several cognitive facets, including attention, working memory, vigilant sensory encoding, rehearsal processing, and behavioral flexibility (Awh and Jonides, 2001; Floresco et al., 2009). In fact, during the delay period of a short-term memory task, the proportion of PFC neuronal activity related to attention is higher than that of memory function (Lebedev et al., 2004). Here, PFC theta power in ELS rats was persistently elevated during the entire length of the trial, 

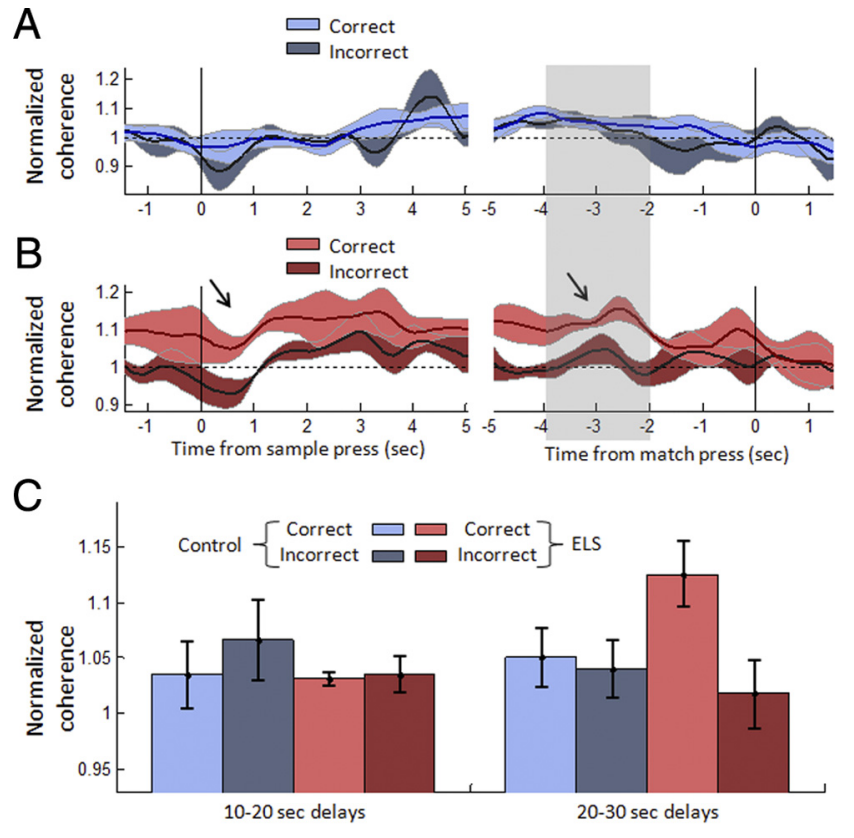

Figure 8. Dynamic CA1-PFC theta coherence in correct and incorrect trials. $A$, In trials with 20 -30 s delays, control rats did not show performance-related differences in CA1-PFC coherence between correct (light blue) and incorrect (dark blue). $\boldsymbol{B}$, In trials with 20 -30 s delays, ELS rats showed increased CA1-PFC coherence in correct trials (light red) relative to incorrect trials (dark red), particularly around the time of the sample press and before the match press (arrows). $\boldsymbol{C}$, CA1-PFC theta coherence during retrieval $(\boldsymbol{A}, \boldsymbol{B}$, gray box) as a function of delay (all blocks included). The data are grouped into two delay levels, and are separated into correct and incorrect trials. Confirming $\boldsymbol{A}$ and $\boldsymbol{B}$, CA1-PFC coherence is increased in correct trials compared with incorrect trials in trials with longer delays among ELS animals but not controls, suggesting that hippocampal-PFC interplay is particularly useful for this group when memory demand is high.

suggesting it may reflect increased attention during active performance. This pattern is similar to activation profiles of the dorsolateral PFC in humans during the delay of a working memory task, attributed to both memory and attention processes in this region (Curtis and D'Esposito, 2003). Thus, the functional significance of enhanced PFC neural activity observed in this study likely involves an array of interplaying cognitive faculties during the execution of the DNMS task, including attention and working memory (Matzel and Kolata, 2010).

Regardless of putative cognitive strategies, distinct oscillation patterns between ELS and control rats during trials demonstrate different neural coordination to solve the task following a neurological insult. These disparities could reflect a compensation between multiple limbic (e.g., hippocampus) and neocortical (e.g., PFC) structures via neural plasticity (Bach-y-Rita, 1990), revealed here by oscillatory network activity (Voytek et al., 2010) in the hippocampal-PFC network. This is particularly supported by the observation that these patterns developed in response to DNMS training and by the relation between accuracy and heightened CA1-PFC theta activity in ELS animals but not among controls.

Overall, our findings demonstrate that following a neurological insult early in life learning and performance may become mediated through alternate yet equally effective neural oscillation pathways, even in fully mature animals. Compensatory neural strategies could be capitalized upon to accelerate cognitive rehabilitation in neurological disorders (Mateer et al., 1996; Stiles et al., 1997). For example, our data suggest that individuals who experienced ELS and have chronic memory impairments may benefit from cognitive training on PFC-mediated functions (e.g., working memory and attentional elements) to aid short-term memory. ELS affect $4 \%$ of the population, and nearly $70 \%$ of these individuals go on to develop cognitive impairment later in life (Legido et al., 1991; Hauser, 1995), indicating that a substantial number of individuals could benefit from these types of tailored interventions. Our study encourages the investigation of alternate neural strategies in other models of neurological disease, using oscillatory activity as an effective surrogate for network activity (Adhikari et al., 2010; Sigurdsson et al., 2010; Voytek et al., 2010). This avenue will shed light on other brain networks that could compensate for stereotyped impairments, and could provide the neurophysiological basis for directed cognitive rehabilitation programs to accelerate and augment cognitive recovery.

\section{References}

Adhikari A, Topiwala MA, Gordon JA (2010) Synchronized activity between the ventral hippocampus and the medial prefrontal cortex during anxiety. Neuron 65:257-269.

Awh E, Jonides J (2001) Overlapping mechanisms of attention and spatial working memory. Trends Cogn Sci 5:119-126.

Bach-y-Rita P (1990) Brain plasticity as a basis for recovery of function in humans. Neuropsychologia 28:547-554.

Benchenane K, Peyrache A, Khamassi M, Tierney PL, Gioanni Y, Battaglia FP, Wiener SI (2010) Coherent theta oscillations and reorganization of spike timing in the hippocampal-prefrontal network upon learning. Neuron 66:921-936.

Beshel J, Kopell N, Kay LM (2007) Olfactory bulb gamma oscillations are enhanced with task demands. J Neurosci 27:8358-8365.

Brown PL, Jenkins HM (1968) Auto-shaping of the pigeon's key-peck. J Exp Anal Behav 11:1-8.

Canolty RT, Edwards E, Dalal SS, Soltani M, Nagarajan SS, Kirsch HE, Berger MS, Barbaro NM, Knight RT (2006) High gamma power is phaselocked to theta oscillations in human neocortex. Science 313:1626-1628.

Carmichael ST, Wei L, Rovainen CM, Woolsey TA (2001) New patterns of intracortical projections after focal cortical stroke. Neurobiol Dis 8:910-922.

Chauvière L, Rafrafi N, Thinus-Blanc C, Bartolomei F, Esclapez M, Bernard C (2009) Early deficits in spatial memory and theta rhythm in experimental temporal lobe epilepsy. J Neurosci 29:5402-5410.

Colgin LL, Moser EI (2010) Gamma oscillations in the hippocampus. Physiology (Bethesda) 25:319-329.

Colgin LL, Denninger T, Fyhn M, Hafting T, Bonnevie T, Jensen O, Moser MB, Moser EI (2009) Frequency of gamma oscillations routes flow of information in the hippocampus. Nature 462:353-357.

Cornejo BJ, Mesches MH, Coultrap S, Browning MD, Benke TA (2007) A single episode of neonatal seizures permanently alters glutamatergic synapses. Ann Neurol 61:411-426.

Curtis CE, D'Esposito M (2003) Persistent activity in the prefrontal cortex during working memory. Trends Cogn Sci 7:415-423.

de Rogalski Landrot I, Minokoshi M, Silveira DC, Cha BH, Holmes GL (2001) Recurrent neonatal seizures: relationship of pathology to the electroencephalogram and cognition. Brain Res Dev Brain Res 129:27-38.

DeCoteau WE, Thorn C, Gibson DJ, Courtemanche R, Mitra P, Kubota Y, Graybiel AM (2007) Learning-related coordination of striatal and hippocampal theta rhythms during acquisition of a procedural maze task. Proc Natl Acad Sci U S A 104:5644-5649.

Feldman DE (2009) Synaptic mechanisms for plasticity in neocortex. Annu Rev Neurosci 32:33-55.

Floresco SB, Zhang Y, Enomoto T (2009) Neural circuits subserving behavioral flexibility and their relevance to schizophrenia. Behav Brain Res 204:396-409.

Gathercole SE, Alloway TP (2006) Practitioner review: short-term and working memory impairments in neurodevelopmental disorders: diagnosis and remedial support. J Child Psychol Psychiatry 47:4-15.

Grinsted A, Moore JC, Jevrejeva S (2004) Application of the cross wavelet transform and wavelet coherence to geophysical time series. Nonlin Processes Geophys 11:561-566.

Hampson RE, Deadwyler SA (1996) Ensemble codes involving hippocam- 
pal neurons are at risk during delayed performance tests. Proc Natl Acad Sci U S A 93:13487-13493.

Hampson RE, Heyser CJ, Deadwyler SA (1993) Hippocampal cell firing correlates of delayed-match-to-sample performance in the rat. Behav Neurosci 107:715-739.

Hampson RE, Jarrard LE, Deadwyler SA (1999) Effects of ibotenate hippocampal and extrahippocampal destruction on delayed-match and -nonmatch-to-sample behavior in rats. J Neurosci 19:1492-1507.

Hauser WA (1995) Epidemiology of epilepsy in children. Neurosurg Clin N Am 6:419-429.

Holmes GL (2009) The long-term effects of neonatal seizures. Clin Perinatol 36:901-914, vii-viii.

Hyman JM, Zilli EA, Paley AM, Hasselmo ME (2005) Medial prefrontal cortex cells show dynamic modulation with the hippocampal theta rhythm dependent on behavior. Hippocampus 15:739-749.

Hyman JM, Zilli EA, Paley AM, Hasselmo ME (2010) Working memory performance correlates with prefrontal-hippocampal theta interactions but not with prefrontal neuron firing rates. Front Integr Neurosci 4:2.

Isaeva E, Isaev D, Khazipov R, Holmes GL (2006) Selective impairment of GABAergic synaptic transmission in the flurothyl model of neonatal seizures. Eur J Neurosci 23:1559-1566.

Joel D, Weiner I, Feldon J (1997) Electrolytic lesions of the medial prefrontal cortex in rats disrupt performance on an analog of the Wisconsin Card Sorting Test, but do not disrupt latent inhibition: implications for animal models of schizophrenia. Behav Brain Res 85:187-201.

Jones MW, Wilson MA (2005) Theta rhythms coordinate hippocampalprefrontal interactions in a spatial memory task. PLoS Biol 3:e402.

Karnam HB, Zhao Q, Shatskikh T, Holmes GL (2009a) Effect of age on cognitive sequelae following early life seizures in rats. Epilepsy Res $85: 221-230$

Karnam HB, Zhou JL, Huang LT, Zhao Q, Shatskikh T, Holmes GL (2009b) Early life seizures cause long-standing impairment of the hippocampal map. Exp Neurol 217:378-387.

Keller TA, Just MA (2009) Altering cortical connectivity: remediationinduced changes in the white matter of poor readers. Neuron 64:624-631.

Kirkby DL, Higgins GA (1998) Characterization of perforant path lesions in rodent models of memory and attention. Eur J Neurosci 10:823-838.

Kleen JK, Scott RC, Holmes GL, Lenck-Santini PP (2010) Hippocampal interictal spikes disrupt cognition in rats. Ann Neurol 67:250-257.

Kojima S, Goldman-Rakic PS (1982) Delay-related activity of prefrontal neurons in rhesus monkeys performing delayed response. Brain Res 248:43-49.

Lebedev MA, Messinger A, Kralik JD, Wise SP (2004) Representation of attended versus remembered locations in prefrontal cortex. PLoS Biol 2:e365.

Lee I, Kesner RP (2003) Time-dependent relationship between the dorsal hippocampus and the prefrontal cortex in spatial memory. J Neurosci 23:1517-1523.

Legido A, Clancy RR, Berman PH (1991) Neurologic outcome after electroencephalographically proven neonatal seizures. Pediatrics 88:583-596.

Marcelin B, Chauvière L, Becker A, Migliore M, Esclapez M, Bernard C (2009) h channel-dependent deficit of theta oscillation resonance and phase shift in temporal lobe epilepsy. Neurobiol Dis 33:436-447.

Markowitsch HJ, Kessler J, Streicher M (1985) Consequences of serial cortical, hippocampal, and thalamic lesions and of different lengths of overtraining on the acquisition and retention of learning tasks. Behav Neurosci 99:233-256.
Mateer CA, Kerns KA, Eso KL (1996) Management of attention and memory disorders following traumatic brain injury. J Learn Disabil 29:618-632.

Matzel LD, Kolata S (2010) Selective attention, working memory, and animal intelligence. Neurosci Biobehav Rev 34:23-30.

McFarland WL, Teitelbaum H, Hedges EK (1975) Relationship between hippocampal theta activity and running speed in the rat. J Comp Physiol Psychol 88:324-328.

Merabet LB, Pascual-Leone A (2010) Neural reorganization following sensory loss: the opportunity of change. Nat Rev Neurosci 11:44-52.

Mitra P, Bokil H (2008) Observed brain dynamics. Oxford, New York: Oxford UP.

Montgomery SM, Buzsáki G (2007) Gamma oscillations dynamically couple hippocampal CA3 and CA1 regions during memory task performance. Proc Natl Acad Sci U S A 104:14495-14500.

Montgomery SM, Betancur MI, Buzsáki G (2009) Behavior-dependent coordination of multiple theta dipoles in the hippocampus. J Neurosci 29:1381-1394.

Mormann F, Fell J, Axmacher N, Weber B, Lehnertz K, Elger CE, Fernández G (2005) Phase/amplitude reset and theta-gamma interaction in the human medial temporal lobe during a continuous word recognition memory task. Hippocampus 15:890-900.

Paxinos G, Watson C (2005) The rat brain in stereotaxic coordinates. San Diego: Academic.

Pych JC, Chang Q, Colon-Rivera C, Haag R, Gold PE (2005) Acetylcholine release in the hippocampus and striatum during place and response training. Learn Mem 12:564-572.

Rizzuto DS, Madsen JR, Bromfield EB, Schulze-Bonhage A, Kahana M) (2006) Human neocortical oscillations exhibit theta phase differences between encoding and retrieval. Neuroimage 31:1352-1358.

Siapas AG, Lubenov EV, Wilson MA (2005) Prefrontal phase locking to hippocampal theta oscillations. Neuron 46:141-151.

Sigurdsson T, Stark KL, Karayiorgou M, Gogos JA, Gordon JA (2010) Impaired hippocampal-prefrontal synchrony in a genetic mouse model of schizophrenia. Nature 464:763-767.

Stiles J, Trauner D, Engel M, Nass R (1997) The development of drawing in children with congenital focal brain injury: evidence for limited functional recovery. Neuropsychologia 35:299-312.

Tsanov M, Manahan-Vaughan D (2009) Long-term plasticity is proportional to theta-activity. PLoS One 4:e5850.

Voytek B, Davis M, Yago E, Barceló F, Vogel EK, Knight RT (2010) Dynamic neuroplasticity after human prefrontal cortex damage. Neuron 68:401408.

Wall PM, Messier C (2001) The hippocampal formation-orbitomedial prefrontal cortex circuit in the attentional control of active memory. Behav Brain Res 127:99-117.

Wang GW, Cai JX (2006) Disconnection of the hippocampal-prefrontal cortical circuits impairs spatial working memory performance in rats. Behav Brain Res 175:329-336.

Wang XJ (2010) Neurophysiological and computational principles of cortical rhythms in cognition. Physiol Rev 90:1195-1268.

Watrous AJ, Fried I, Ekstrom AD (2011) Behavioral correlates of human hippocampal delta and theta oscillations during navigation. J Neurophysiol 105:1747-1755.

Zhou X, Merzenich MM (2009) Developmentally degraded cortical temporal processing restored by training. Nat Neurosci 12:26-28. 